\title{
Long Time Decay Rate to a Bipolar Quantum Drift-Diffusion Model
}

\author{
Yingjie Zhu, ${ }^{1,2,3}$ Bo Liang, ${ }^{3}$ and Xiting Peng ${ }^{3}$ \\ ${ }^{1}$ Institute of Mathematics, Jilin University, Changchun 130012, China \\ ${ }^{2}$ College of Science, Changchun University, Changchun 130022, China \\ ${ }^{3}$ School of Science, Dalian Jiaotong University, Dalian 116028, China \\ Correspondence should be addressed to Yingjie Zhu; yingjiezhu2012@163.com
}

Received 31 January 2015; Accepted 12 April 2015

Academic Editor: Ajda Fošner

Copyright (C) 2015 Yingjie Zhu et al. This is an open access article distributed under the Creative Commons Attribution License, which permits unrestricted use, distribution, and reproduction in any medium, provided the original work is properly cited.

This paper is devoted to studying the long time behavior of solutions to a bipolar quantum hydrodynamic in one-dimensional space for general pressure functions. The model is usually applied to simulate some quantum effects in semiconductor devices. The decay rate for time variable is obtained by the entropy functional method and semidiscrete technique.

\section{Introduction}

By performing relaxation time limit in the quantum hydrodynamic equation, the semiconductor quantum drift-diffusion model can be obtained. Usually, it is applied to simulate the quantum effects, for example, resonant tunneling in semiconductor devices. Formally, the model also belongs to the field of the fourth-order parabolic equations (see [1]) including the thin film equation (see [2-4]) and the CahnHilliard equation. In the paper, we are mainly concerned with the following bipolar quantum drift-diffusion model in onedimensional space:

$$
\begin{aligned}
& n_{t}+\frac{\varepsilon^{2}}{2}\left(n(\log n)_{x x}\right)_{x x}-\left(P_{n}(n)\right)_{x x}+\left(n V_{x}\right)_{x}=0, \\
& p_{t}+\frac{\varepsilon^{2}}{2}\left(p(\log p)_{x x}\right)_{x x}-\left(P_{p}(p)\right)_{x x}-\left(p V_{x}\right)_{x}=0, \\
& \lambda^{2} V_{x x}=n-p-C(x), \quad(x, t) \in Q_{T}=\Omega \times(0, T)
\end{aligned}
$$

with the initial-boundary conditions as follows:

$$
\begin{gathered}
n=p=1, \\
n_{x}=p_{x}=0,
\end{gathered}
$$

$$
\begin{aligned}
V & =V_{D}, \\
n(\cdot, 0) & =n_{0}, \\
p(\cdot, 0) & =p_{0},
\end{aligned}
$$

in $\Omega$,

where $\Omega=(0,1), V_{D}$ is a constant, $n$ is the electron density, $p$ is the positively charged ion (or hole) density, and $V$ is the electron static potential. $P_{n}$ and $P_{p}$ are the pressure functions and the function $C(x)$ is the doping profile. The parameter $\varepsilon$ is the scaled Plank constant and $\lambda>0$ is the Debye length.

Dolbeault et al. [1] studied the existence and uniqueness of the fourth-order parabolic equation $n_{t}+\left(n(\log n)_{x x}\right)_{x x}=0$ with periodic boundary conditions. For the same equation, Jüngel and Toscani [5] used the entropy functional method and the semidiscrete technique to construct an iteration and obtained the exponential decay results. By employing the semidiscrete method, Jüngel and Violet obtained the existence of weak solution and gave the quasineutral limit in [6] to the bipolar quantum drift-diffusion model.

Generally, the bipolar model is more meaningful in physics and we will treat the case with a general pressure 
function. By applying the entropy method (see [7]) and iteration procedure which have already been used successfully in [5], we will get the long time exponential decay rate to the quantum drift-diffusion model (1)-(4). It is a key to deal with the coupling relationship in the Poisson equation (3). Moreover, since the maximum principle does not hold again for the high order partial differential equations, we need to overcome this difficulty for the purpose of getting uniform energy estimates.

As [6] has shown, by letting $\tau=T / n, n_{k}=n(x, k h), p_{k}=$ $p(x, k h)$, and $k=1,2, \ldots, n$, we still borrow the semidiscrete system. Consider

$$
\begin{aligned}
& \frac{1}{\tau}\left(n_{k}-n_{k-1}\right)+\frac{\varepsilon^{2}}{2}\left(n_{k}\left(\log n_{k}\right)_{x x}\right)_{x x}-\left(P_{n}\left(n_{k}\right)\right)_{x x} \\
& \quad+\left(n_{k} V_{k x}\right)_{x}=0, \\
& \frac{1}{\tau}\left(p_{k}-p_{k-1}\right)+\frac{\varepsilon^{2}}{2}\left(p_{k}\left(\log p_{k}\right)_{x x}\right)_{x x}-\left(P_{p}\left(p_{k}\right)\right)_{x x} \\
& \quad-\left(p_{k} V_{k x}\right)_{x}=0, \\
& \lambda^{2} V_{k x x}=n_{k}-p_{k}-C(x), \quad \text { in } \Omega, \\
& n_{k}=p_{k}=1, \\
& n_{k x}=p_{k x}=0, \\
& V_{k}=V_{D},
\end{aligned}
$$

on $\partial \Omega$.

For the problem (1)-(4) and the semidiscrete system (5)-(8), we list some results (Theorems 1-3) which had been proved in $[6]$.

Theorem 1. Assume $C(x) \in L^{\infty}(\Omega), 0 \leq n_{0}, p_{0} \in L^{1}(\Omega)$, and

$$
\begin{aligned}
\int_{\Omega}\left(n_{0}-\log n_{0}\right) d x+\int_{\Omega}\left(p_{0}-\log p_{0}\right) d x \\
\quad+\int_{\Omega}\left(n_{0}\left(\log n_{0}-1\right)+1\right) d x \\
\quad+\int_{\Omega}\left(p_{0}\left(\log p_{0}-1\right)+1\right) d x<\infty .
\end{aligned}
$$

Let $P_{i} \in C^{1}[0, \infty)(i=n, p)$ be nondecreasing and assume that there exist two constants $0<q<7 / 2$ and $C_{P}>0$ such that $P_{i}(x) \leq C_{P}\left(1+|x|^{q}\right)$ for $x \geq 0, i=n, p$. Then there exists $a$ weak solution $(n, p, V)$ to (1)-(4) such that

$$
\begin{gathered}
n, p \geq 0, \quad \text { in } Q_{T}, \\
n, p \in L^{7 / 2}\left(Q_{T}\right), \\
\log n, \log p \in L^{2}\left(0, T ; H_{0}^{2}(\Omega)\right), \\
n_{t}, p_{t} \in L^{1}\left(0, T ; H^{-3}(\Omega)\right), \\
V \in L^{\infty}\left(0, T ; H^{2}(\Omega)\right) .
\end{gathered}
$$

Theorem 2. Under the assumptions of Theorem 1, there exists a weak solution $\left(n_{k}, p_{k}, V_{k}\right) \in H^{2}(\Omega) \times H^{2}(\Omega) \times H^{2}(\Omega)$ of $(5)-$ (8) satisfying $\left(\log n_{k}, \log p_{k}, V_{k}-V_{D}\right) \in H_{0}^{2}(\Omega) \times H_{0}^{2}(\Omega) \times H_{0}^{1}(\Omega)$ and $n_{k}, p_{k}>0$ in $\Omega$.

By defining the approximate solutions

$$
\begin{aligned}
& n^{(\tau)}(x, t)=n_{k}(x), \\
& \widetilde{n^{(\tau)}}(x, t)=n_{k-1}(x), \\
& p^{(\tau)}(x, t)=p_{k}(x), \\
& \widetilde{p^{(\tau)}}(x, t)=p_{k-1}(x), \\
& V^{(\tau)}(x, t)=V_{k}(x)
\end{aligned}
$$

for $x \in \Omega, t \in((k-1) \tau, k \tau]$, [6] gave the following convergence results.

Theorem 3. Under the assumptions of Theorem 1, there exists a subsequence of $\left(n^{(\tau)}, p^{(\tau)}, V^{(\tau)}\right)$ (not relabeled) such that

$$
\begin{aligned}
& \log n^{(\tau)} \rightarrow \log n \\
& \log p^{(\tau)}-\log p,
\end{aligned}
$$

weakly in $L^{2}\left(0, T ; H^{2}(\Omega)\right)$,

$$
\begin{aligned}
& n^{(\tau)} \longrightarrow n, \\
& p^{(\tau)} \longrightarrow p, \\
& \text { strongly in } L^{2}\left(Q_{T}\right), \\
& P_{n}\left(n^{(\tau)}\right) \longrightarrow P_{n}(n), \\
& P_{p}\left(p^{(\tau)}\right) \longrightarrow P_{p}(p), \\
& \frac{1}{\tau}\left(n^{(\tau)}-\widetilde{n^{(\tau)}}\right) \rightarrow n_{t}, \quad \text { weakly in } L^{s}\left(0, T ; H^{-3}(\Omega)\right), \\
& \frac{1}{\tau}\left(p^{(\tau)}-\widetilde{p^{(\tau)}}\right) \rightarrow p_{t}, \quad \\
& V^{(\tau)} \rightarrow V, \quad \text { weakly in } L^{2}\left(0, T ; H^{1}(\Omega)\right)
\end{aligned}
$$

and it holds

$$
\begin{aligned}
\int_{0}^{T}\left\langle\partial_{t} n, \phi\right\rangle d t= & -\iint_{Q_{T}} n(\log n)_{x x} \phi_{x x} d x d t \\
& +\iint_{Q_{T}} P_{n}(n) \phi_{x x} d x d t \\
& +\iint_{Q_{T}} n V_{x} \phi_{x} d x d t
\end{aligned}
$$




$$
\begin{aligned}
\int_{0}^{T}\left\langle\partial_{t} p, \phi\right\rangle d t= & -\iint_{\mathrm{Q}_{T}} p(\log p)_{x x} \phi_{x x} d x d t \\
& +\iint_{\mathrm{Q}_{T}} P_{p}(p) \phi_{x x} d x d t \\
& -\iint_{\mathrm{Q}_{T}} p V_{x} \phi_{x} d x d t, \\
\lambda^{2} \iint_{\mathrm{Q}_{T}} V_{x} \phi_{x} d x d t= & -\iint_{\mathrm{Q}_{T}}(n-p) \phi d x d t
\end{aligned}
$$

for all $\phi \in C_{0}^{\infty}\left(Q_{T}\right)$, where $s=\min \{7 / 2 q, 14 / 11\}>1$ and $\langle\cdot, \cdot\rangle$ is the duality product between $H^{-3}(\Omega)$ and $H_{0}^{3}(\Omega)$.

The main result of the paper is as follows.

Theorem 4. Under the assumptions of Theorem 1, let $C(x) \equiv 0$ and let $(n, p, V)$ be the weak solution to (1)-(4). Then

$$
\begin{aligned}
& \|n(t)-1\|_{L^{1}(\Omega)}+\|p(t)-1\|_{L^{1}(\Omega)}+\left\|V(t)-V_{D}\right\|_{H^{1}(\Omega)} \\
& \quad \leq C_{1}\left(\eta_{0}+\xi_{0}\right) e^{-C_{2} t}
\end{aligned}
$$

for $t \geq 0$, where the constants $C_{1}$ and $C_{2}>0$ only depend on $\Omega, n_{0}$, and $p_{0}$.

Here, we need the condition $C(x) \equiv 0$ for the purpose of integration by parts and nonpositivity for some terms.

The paper is arranged as follows. We will prove some auxiliary lemmas at first in Section 2. The exponential decay rate will be established in Section 3 .

\section{Semidiscrete Solutions}

Introduce some discrete entropies

$$
\begin{aligned}
\eta_{k} & =\int_{\Omega}\left(n_{k}\left(\log n_{k}-1\right)+1\right) d x, \\
\xi_{k} & =\int_{\Omega}\left(p_{k}\left(\log p_{k}-1\right)+1\right) d x, \\
q_{k} & =\int_{\Omega}\left(n_{k}-\log n_{k}\right) d x, \\
\zeta_{k} & =\int_{\Omega}\left(p_{k}-\log p_{k}\right) d x,
\end{aligned}
$$

for $k \in N$. For the positive entropies $\eta_{k}$ and $\xi_{k}$, we have the following iteration estimate.

Lemma 5. Assume $\eta_{k-1}, \xi_{k-1}<\infty, k \geq 1$. Then

$$
\begin{aligned}
\frac{1}{\tau}\left(\eta_{k}-\eta_{k-1}\right)+\frac{1}{\tau}\left(\xi_{k}-\xi_{k-1}\right)+\frac{\varepsilon^{2}}{2} \int_{\Omega} n_{k}\left(\log n_{k}\right)_{x x}^{2} d x \\
+\frac{\varepsilon^{2}}{2} \int_{\Omega} p_{k}\left(\log p_{k}\right)_{x x}^{2} d x \leq 0 .
\end{aligned}
$$

Proof. Multiplying (5) and (6) by $\log n_{k}$ and $\log p_{k}$, respectively, we have

$$
\begin{aligned}
& \frac{1}{\tau} \int_{\Omega}\left(n_{k}-n_{k-1}\right) \log n_{k} d x+\frac{\varepsilon^{2}}{2} \int_{\Omega} n_{k}\left(\log n_{k}\right)_{x x}^{2} d x \\
& \quad=-\int_{\Omega}\left(\left(P_{n}\left(n_{k}\right)\right)_{x}-n_{k} V_{k x}\right)\left(\log n_{k}\right)_{x} d x, \\
& \frac{1}{\tau} \int_{\Omega}\left(p_{k}-p_{k-1}\right) \log p_{k} d x+\frac{\varepsilon^{2}}{2} \int_{\Omega} p_{k}\left(\log p_{k}\right)_{x x}^{2} d x \\
& \quad=-\int_{\Omega}\left(\left(P_{p}\left(p_{k}\right)\right)_{x}+p_{k} V_{k x}\right)\left(\log p_{k}\right)_{x} d x .
\end{aligned}
$$

A direct calculation yields

$$
-\int_{\Omega}\left(P_{n}\left(n_{k}\right)\right)_{x}\left(\log n_{k}\right)_{x} d x=-\int_{\Omega} P_{n}^{\prime}\left(n_{k}\right) \frac{n_{k x}^{2}}{n_{k}} d x \leq 0
$$

and similarly $-\int_{\Omega}\left(P_{p}\left(p_{k}\right)\right)_{x}\left(\log p_{k}\right)_{x} d x \leq 0$. On the other hand, a simple calculation gives

$$
\int_{\Omega} n_{k} V_{k x}\left(\log n_{k}\right)_{x} d x=\int_{\Omega} V_{k x} n_{k x} d x,
$$

and similarly $-\int_{\Omega} p_{k} V_{k x}\left(\log p_{k}\right)_{x} d x=-\int_{\Omega} V_{k x} p_{k x} d x$. Letting $n_{k}-p_{k}$ be a test function in the Poisson equation (7), we get

$$
\lambda^{2} \int_{\Omega} V_{k x}\left(n_{k}-p_{k}\right)_{x} d x=-\int_{\Omega}\left(n_{k}-p_{k}\right)^{2} d x \leq 0 .
$$

Combining (18)-(20) with (17), we have

$$
\begin{aligned}
\frac{1}{\tau} \int_{\Omega} & \left(n_{k}-n_{k-1}\right) \log n_{k} d x+\frac{\varepsilon^{2}}{2} \int_{\Omega} n_{k}\left(\log n_{k}\right)_{x x}^{2} d x \\
+ & \frac{1}{\tau} \int_{\Omega}\left(p_{k}-p_{k-1}\right) \log p_{k} d x \\
+ & \frac{\varepsilon^{2}}{2} \int_{\Omega} p_{k}\left(\log p_{k}\right)_{x x}^{2} d x \leq 0 .
\end{aligned}
$$

Furthermore, the inequality $-\log x+x-1 \geq 0$ for all $x>0$ implies

$$
\begin{aligned}
\frac{1}{\tau} \int_{\Omega}\left(n_{k}-n_{k-1}\right) \log n_{k} d x \\
=\frac{1}{\tau}\left(\eta_{k}-\eta_{k-1}\right) \\
\quad+\frac{1}{\tau} \int_{\Omega} n_{k-1}\left(-\log \frac{n_{k}}{n_{k-1}}+\frac{n_{k}}{n_{k-1}}-1\right) d x \\
\quad \geq \frac{1}{\tau}\left(\eta_{k}-\eta_{k-1}\right)
\end{aligned}
$$

and similarly $(1 / \tau) \int_{\Omega}\left(p_{k}-p_{k-1}\right) \log p_{k} d x \geq(1 / \tau)\left(\xi_{k}-\right.$ $\left.\xi_{k-1}\right)$. Now we have completed the proof. 
Lemma 6. Assume $q_{k-1}, \zeta_{k-1}<\infty, k \geq 1$. Then

$$
\begin{aligned}
& \frac{1}{\tau}\left(q_{k}-q_{k-1}\right)+\frac{\varepsilon^{2}}{2} \int_{\Omega}\left(\log n_{k}\right)_{x x}^{2} d x+\frac{1}{\tau}\left(\zeta_{k}-\zeta_{k-1}\right) \\
& +\frac{\varepsilon^{2}}{2} \int_{\Omega}\left(\log p_{k}\right)_{x x}^{2} d x \leq 0 .
\end{aligned}
$$

Proof. Multiply (5) and (6) by $1-1 / n_{k}$ and $1-1 / p_{k}$, respectively, to get

$$
\begin{aligned}
\frac{1}{\tau} \int_{\Omega} & \left(n_{k}-n_{k-1}-1+\frac{n_{k-1}}{n_{k}}\right) d x+\frac{\varepsilon^{2}}{2} \int_{\Omega}\left(\log n_{k}\right)_{x x}^{2} d x \\
= & \frac{\varepsilon^{2}}{2} \int_{\Omega}\left(\log n_{k}\right)_{x x}\left(\log n_{k}\right)_{x}^{2} d x-\int_{\Omega} P_{n}^{\prime}\left(n_{k}\right) \frac{n_{k x}^{2}}{n_{k}^{2}} d x \\
& +\int_{\Omega} V_{k x}\left(\log n_{k}\right)_{x} d x \\
= & \frac{\varepsilon^{2}}{2} \int_{\Omega}\left(\left(\log n_{k}\right)_{x}^{3}\right)_{x}^{2} d x-\int_{\Omega} P_{n}^{\prime}\left(n_{k}\right) \frac{n_{k x}^{2}}{n_{k}^{2}} d x \\
& +\int_{\Omega} V_{k x}\left(\log n_{k}\right)_{x} d x \leq \int_{\Omega} V_{k x}\left(\log n_{k}\right)_{x} d x \\
\frac{1}{\tau} \int_{\Omega} & \left(p_{k}-p_{k-1}-1+\frac{p_{k-1}}{p_{k}}\right) d x+\frac{\varepsilon^{2}}{2} \int_{\Omega}\left(\log p_{k}\right)_{x x}^{2} d x \\
\leq & -\int_{\Omega} V_{k x}\left(\log p_{k}\right)_{x} d x .
\end{aligned}
$$

Using $\log n_{k}-\log p_{k}$ as a test function in (7) and applying the inequality $\left(x_{1}-x_{2}\right)\left(\log x_{1}-\log x_{2}\right) \geq 0$ for all $x_{1}, x_{2}>0$, we have

$$
\begin{aligned}
& \lambda^{2} \int_{\Omega} V_{k x}\left(\log n_{k}-\log p_{k}\right)_{x} d x \\
& \quad=-\int_{\Omega}\left(n_{k}-p_{k}\right)\left(\log n_{k}-\log p_{k}\right) d x \leq 0 .
\end{aligned}
$$

The inequality $x-1 \geq \log x$ for $x>0$ yields

$$
\begin{aligned}
& \int_{\Omega}\left(n_{k}-n_{k-1}-1+\frac{n_{k-1}}{n_{k}}\right) d x \\
& \quad=q_{k}-q_{k-1}+\int_{\Omega}\left(-\log \frac{n_{k-1}}{n_{k}}+\frac{n_{k-1}}{n_{k}}-1\right) d x \\
& \quad \geq q_{k}-q_{k-1}
\end{aligned}
$$

and similarly $\int_{\Omega}\left(p_{k}-p_{k-1}-1+p_{k-1} / p_{k}\right) d x \geq \zeta_{k}-\zeta_{k-1}$. By (24)-(27), we obtain

$$
\begin{aligned}
& \frac{1}{\tau}\left(q_{k}-q_{k-1}\right)+\frac{\varepsilon^{2}}{2} \int_{\Omega}\left(\log n_{k}\right)_{x x}^{2} d x+\frac{1}{\tau}\left(\zeta_{k}-\zeta_{k-1}\right) \\
& \quad+\frac{\varepsilon^{2}}{2} \int_{\Omega}\left(\log p_{k}\right)_{x x}^{2} d x \\
& \quad \leq \int_{\Omega} V_{k x}\left(\log n_{k}-\log p_{k}\right)_{x} d x \leq 0
\end{aligned}
$$

Hence, (23) has been proved.
Lemma 7. Assume $q_{0}+\zeta_{0}<\infty$. Then

$$
0<e^{-\left(q_{0}+\zeta_{0}\right)} \leq \int_{\Omega}\left(n_{k}+p_{k}\right) d x \leq 2\left(q_{0}+\zeta_{0}\right)<\infty .
$$

Proof. The inequality $x-\log x \geq x / 2$ for $x>0$ gives

$$
\int_{\Omega}\left(n_{k}+p_{k}\right) d x \leq 2\left(q_{k}+\zeta_{k}\right) \leq 2\left(q_{0}+\zeta_{0}\right)
$$

and Jensen's inequality yields

$$
\begin{aligned}
-\log \int_{\Omega}\left(n_{k}+p_{k}\right) d x \leq & -\int_{\Omega} \log \left(n_{k}+p_{k}\right) d x \\
& +\int_{\Omega}\left(n_{k}+p_{k}\right) d x \\
\leq & \frac{1}{2} \int_{\Omega}\left(n_{k}+p_{k}\right) d x \leq q_{0}+\zeta_{0} \\
& <\infty .
\end{aligned}
$$

The assertion finishes the proof of the lemma.

\section{Exponential Decay}

In order to prove Theorem 4, we list some known results (see [5]) at first.

Lemma 8. Assume the function $u \in H^{2}(\Omega), u>0$, in $\Omega$ and $u=1, u_{x}=0$, on $\partial \Omega$. Then

$$
\int_{\Omega} u(\log u)_{x x}^{2} d x \geq 8\|\sqrt{u}-1\|_{L^{\infty}(\Omega)}^{2}
$$

Lemma 9. Assume the function $u \in L^{\infty}(\Omega), u>0$, in $\Omega$. Then

$$
\begin{aligned}
& \int_{\Omega}(u(\log u-1)+1) d x \\
& \quad \leq\left(\int_{\Omega} u d x+2\right)\|\sqrt{u}-1\|_{L^{\infty}(\Omega)}^{2} .
\end{aligned}
$$

Lemma 10 (Criszar-Kullback-type inequality). Assume the function $0<u \in L^{1}(\Omega)$ and $q=\int_{\Omega}(u-\log u) d x<\infty$. Then

$$
\begin{aligned}
& \int_{\Omega}(u(\log u-1)+1) d x \\
& \geq \frac{1}{(1+\sqrt{2 q})^{2}}\left(\int_{\Omega}|u-1| d x\right)^{2} .
\end{aligned}
$$


Proof of Theorem 4. According to Lemmas 5, 8, 9, and 7, we get

$$
\begin{aligned}
& \left(\eta_{k}-\eta_{k-1}\right)+\left(\xi_{k}-\xi_{k-1}\right) \leq-\frac{\tau}{2} \varepsilon^{2}\left(\int_{\Omega} n_{k}\left(\log n_{k}\right)_{x x}^{2} d x\right. \\
& \left.+\frac{\varepsilon^{2}}{2} \int_{\Omega} p_{k}\left(\log p_{k}\right)_{x x}^{2} d x\right) \\
& \leq-4 \tau \varepsilon^{2}\left(\left\|\sqrt{n_{k}}-1\right\|_{L^{\infty}(\Omega)}^{2}+\left\|\sqrt{p_{k}}-1\right\|_{L^{\infty}(\Omega)}^{2}\right) \\
& \leq-4 \tau \varepsilon^{2}\left(\frac{\eta_{k}}{\int_{\Omega} n_{k} d x+2}+\frac{\xi_{k}}{\int_{\Omega} p_{k} d x+2}\right) \\
& \leq \frac{-2 \tau \varepsilon^{2}}{q_{0}+\zeta_{0}+1}\left(\eta_{k}+\xi_{k}\right)
\end{aligned}
$$

and then, by iterating the above inequality, we deduce that

$$
\begin{aligned}
\eta_{k}+\xi_{k} & \leq\left(1+\frac{2 \tau \varepsilon^{2}}{q_{0}+\zeta_{0}+1}\right)^{-1}\left(\eta_{k-1}+\xi_{k-1}\right) \\
& \leq\left(1+\frac{2 \tau \varepsilon^{2}}{q_{0}+\zeta_{0}+1}\right)^{-k}\left(\eta_{0}+\xi_{0}\right)
\end{aligned}
$$

Moreover, for $(k-1) \tau<t \leq k \tau$, we have

$$
\eta_{k}+\xi_{k} \leq\left(1+\frac{2 \tau \varepsilon^{2}}{q_{0}+\zeta_{0}+1}\right)^{-t / \tau}\left(\eta_{0}+\xi_{0}\right)
$$

Introduce the following functions:

$$
\begin{aligned}
& \eta^{(\tau)}(t)=\int_{\Omega}\left(n^{(\tau)}(t)\left(\log n^{(\tau)}(t)-1\right)+1\right) d x \\
& \xi^{(\tau)}(t)=\int_{\Omega}\left(p^{(\tau)}(t)\left(\log p^{(\tau)}(t)-1\right)+1\right) d x .
\end{aligned}
$$

Equation (37) implies

$$
\eta^{(\tau)}(t)+\xi^{(\tau)}(t) \leq\left(\eta_{0}+\xi_{0}\right)\left(1+\frac{2 \tau \varepsilon^{2}}{q_{0}+\zeta_{0}+1}\right)^{-t / \tau} .
$$

By applying Theorem 3, we conclude that there exists a subsequence of $\left(n^{(\tau)}, p^{(\tau)}\right)$ such that $n^{(\tau)}(t) \rightarrow n$ and $p^{(\tau)}(t) \rightarrow p$ a.e. in $\Omega$. Furthermore, we have $n^{(\tau)} \log n^{(\tau)} \rightarrow$ $n \log n$ and $p^{(\tau)} \log p^{(\tau)} \rightarrow p \log p$ a.e. in $\Omega$. On the other hand, $\eta^{(\tau)}+\xi^{(\tau)}$ is bounded uniformly in $\tau$ from (39) and then Lebesgue's convergence theorem yields $\eta^{(\tau)}+\xi^{(\tau)} \rightarrow \eta+\xi$ for $t \in(0, T)$. Therefore, we have

$$
\begin{aligned}
\eta(t)+\xi(t) & \leq\left(\eta_{0}+\xi_{0}\right) \lim _{\tau \rightarrow 0}\left(1+\frac{2 \tau \varepsilon^{2}}{q_{0}+\zeta_{0}+1}\right)^{-t / \tau} \\
& =\left(\eta_{0}+\xi_{0}\right) e^{-\left(2 /\left(q_{0}+\zeta_{0}+1\right)\right) t}
\end{aligned}
$$

Applying Lemma 10, we have

$$
\|n(t)-1\|_{L^{1}(\Omega)}+\|p(t)-1\|_{L^{1}(\Omega)} \leq C_{1}\left(\eta_{0}+\xi_{0}\right) e^{-C_{2} t},
$$

where $C_{1}$ and $C_{2}$ are both positive constants. Finally, multiply (7) by $V_{k}-V_{D}$ to get

$$
\begin{aligned}
& \lambda^{2} \int_{\Omega}\left|\left(V_{k}-V_{D}\right)_{x}\right|^{2} d x \\
& \quad=-\int_{\Omega}\left(n_{k}-p_{k}\right)\left(V_{k}-V_{D}\right) d x \\
& \quad \leq \int_{\Omega}\left|n_{k}-p_{k}\right| d x\left\|V_{k}-V_{D}\right\|_{L^{\infty}(\Omega)} \\
& \quad \leq C\left\|V_{k}-V_{D}\right\|_{H^{1}(\Omega)} \int_{\Omega}\left|n_{k}-p_{k}\right| d x
\end{aligned}
$$

and then

$$
\lambda^{2}\left\|V^{(\tau)}-V_{D}\right\|_{H^{1}(\Omega)} \leq C \int_{\Omega}\left|n^{(\tau)}-p^{(\tau)}\right| d x .
$$

By letting $\tau \rightarrow 0$, we can get the result of the theorem.

\section{Conflict of Interests}

The authors declare that there is no conflict of interests regarding the publication of this paper.

\section{Acknowledgment}

The authors are grateful to the anonymous referees for useful comments and suggestions which improve the exposition of the paper. This research is supported by the National Natural Science Foundation of China (nos. 11201045 and 11171350), the Scientific and Technological Research project of Jilin Province's Education Department (no. 2013267), the Youth Project of Jilin Province's Science and Technology Department (nos. 20130522099JH, 201201140, and 201115133), and the Twelfth Five-Year Plan project of Jilin Province's Educational Science (no. ZD2014078).

\section{References}

[1] J. Dolbeault, I. Gentil, and A. Jüngel, "A logarithmic fourthorder parabolic equation and related logarithmic Sobolev inequalities," Communications in Mathematical Sciences, vol. 4, no. 2, pp. 275-290, 2006.

[2] F. Bernis and A. Friedman, "Higher order nonlinear degenerate parabolic equations," Journal of Differential Equations, vol. 83, no. 1, pp. 179-206, 1990.

[3] L. Giacomelli, "A fourth-order degenerate parabolic equation describing thin viscous flows over an inclined plane," Applied Mathematics Letters, vol. 12, no. 8, pp. 107-111, 1999.

[4] T. G. Myers, "Thin films with high surface tension," SIAM Review, vol. 40, no. 3, pp. 441-462, 1998.

[5] A. Jüngel and G. Toscani, "Exponential time decay of solutions to a nonlinear fourth-order parabolic equation," Zeitschrift für angewandte Mathematik und Physik ZAMP, vol. 54, no. 3, pp. 377-386, 2003. 
[6] A. Jüngel and I. Violet, "The quasineutral limit in the quantum drift-diffusion equations," Asymptotic Analysis, vol. 53, no. 3, pp. 139-157, 2007.

[7] B. Liang and S. Zheng, "Exponential decay to a quantum hydrodynamic model for semiconductors," Nonlinear Analysis: Real World Applications, vol. 9, no. 2, pp. 326-337, 2008. 


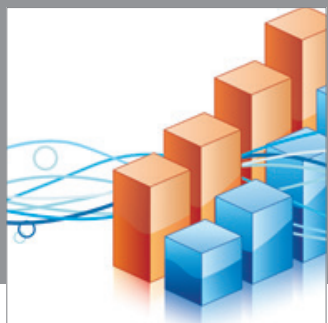

Advances in

Operations Research

mansans

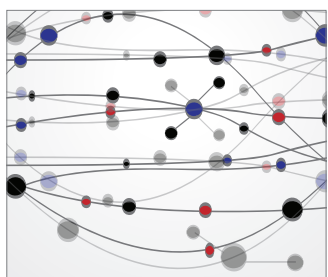

The Scientific World Journal
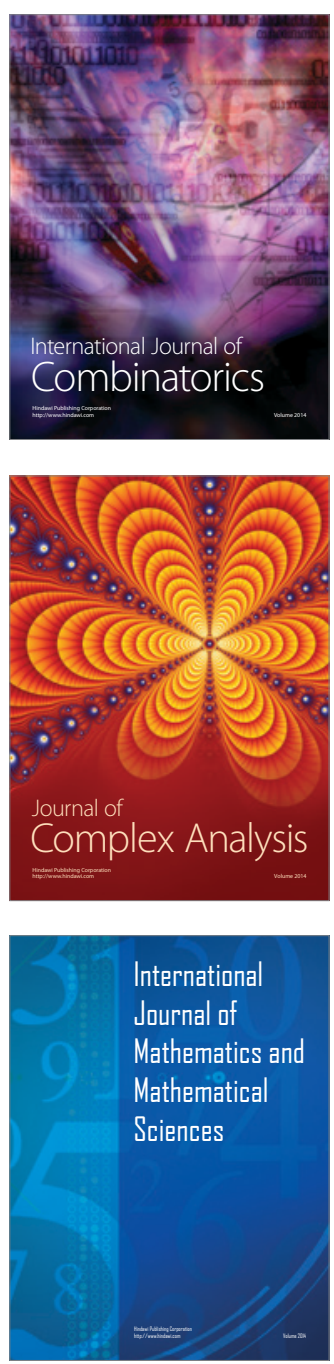
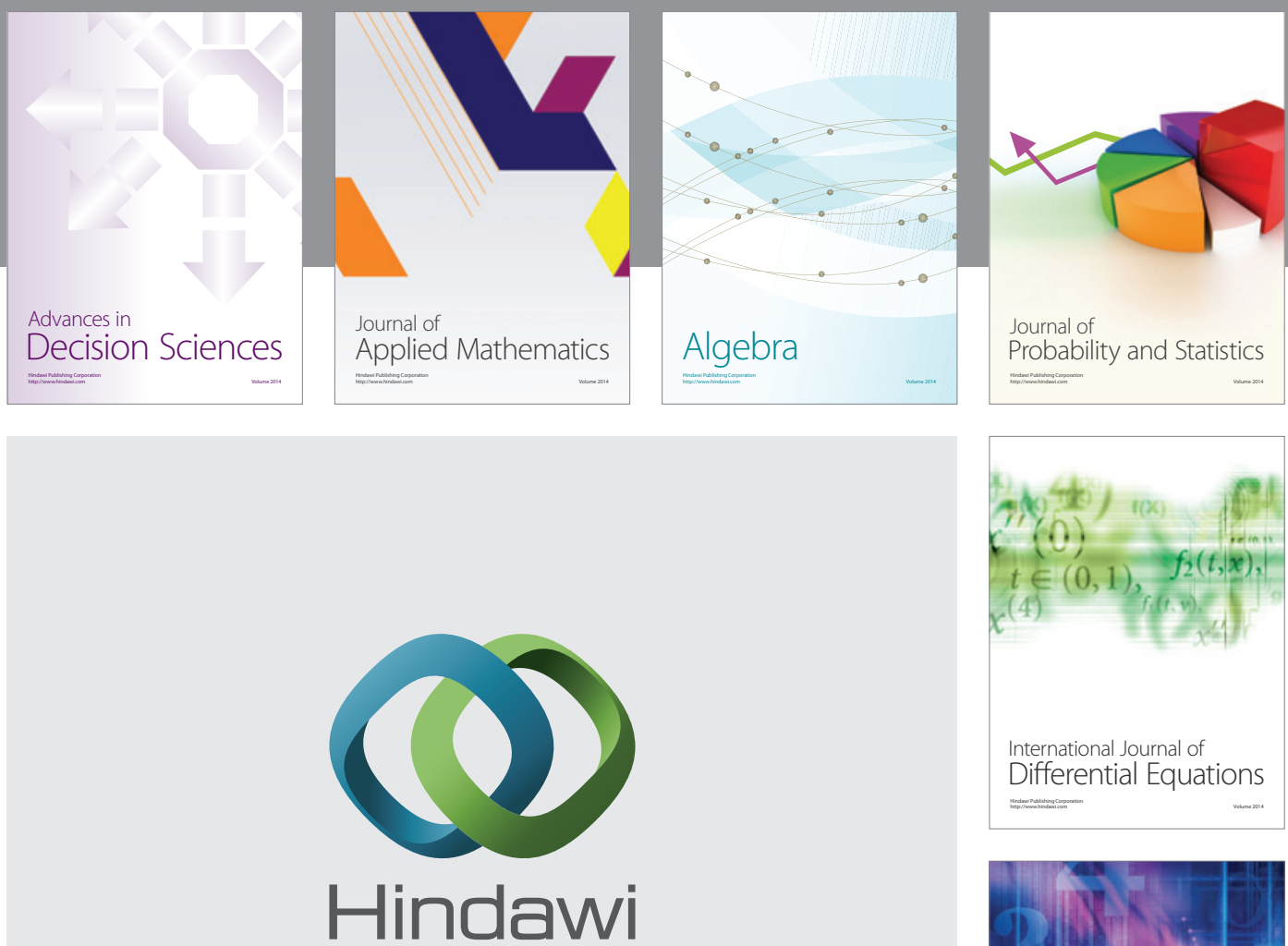

Submit your manuscripts at http://www.hindawi.com
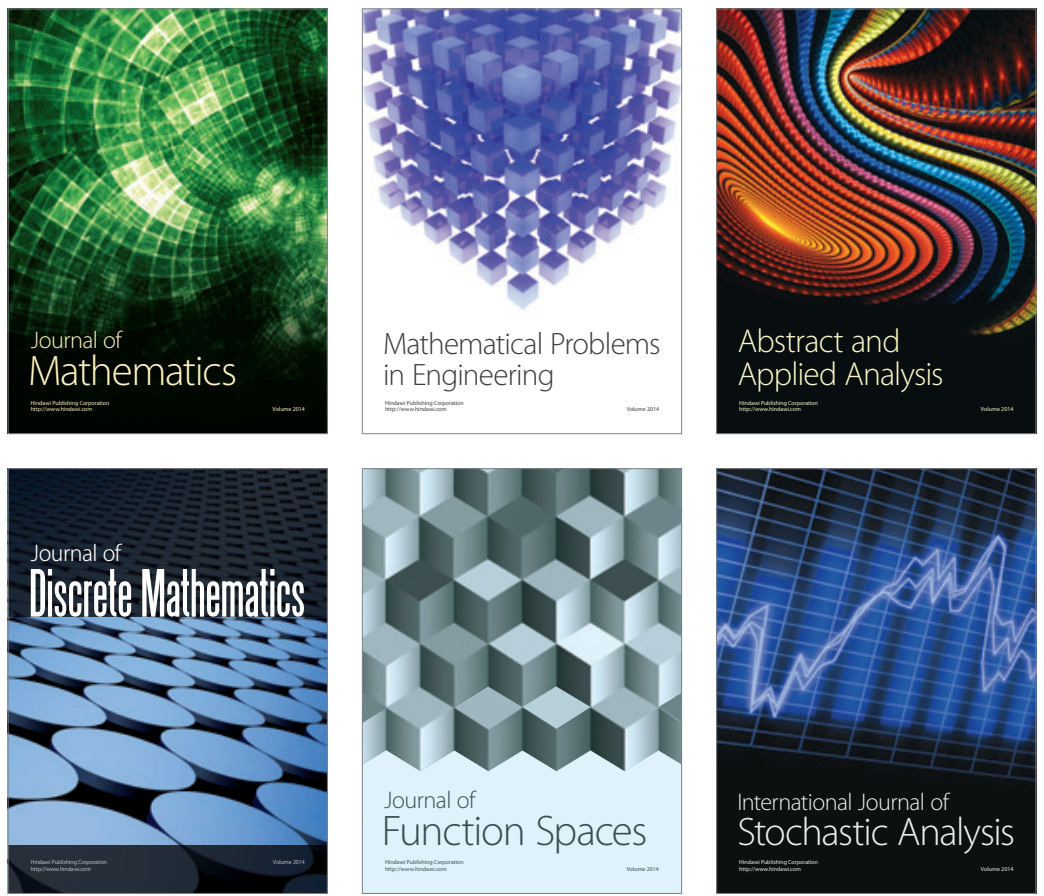

Journal of

Function Spaces

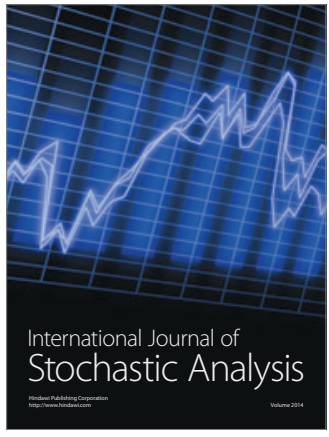

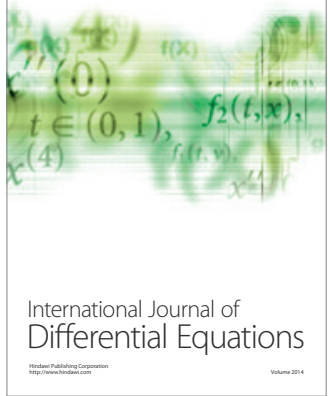
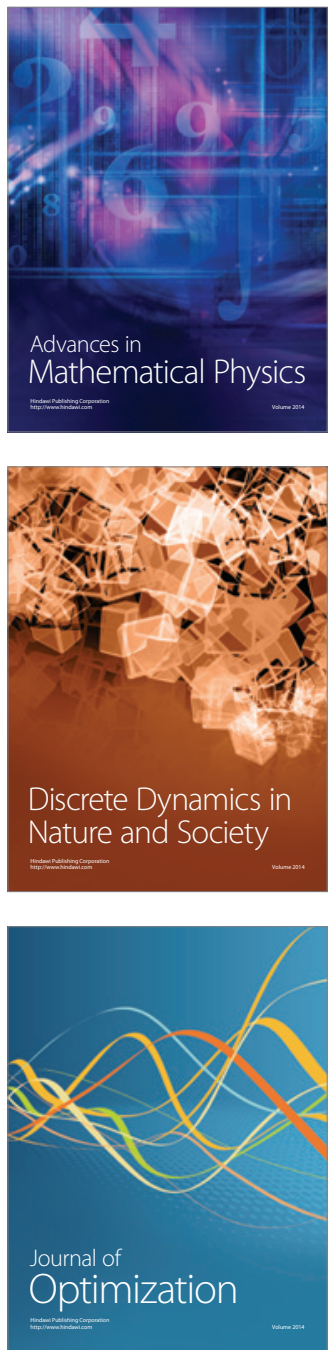